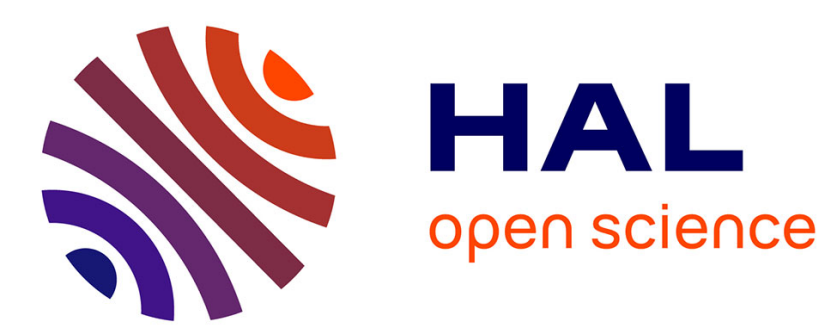

\title{
The Dynamics of a Tippe Top
}

A.C. Or

\section{- To cite this version:}

A.C. Or. The Dynamics of a Tippe Top. SIAM Journal on Applied Mathematics, 1994, 54 (3), pp.597-609. 10.1137/S0036139992235123 . hal-01975393

\section{HAL Id: hal-01975393 \\ https://hal.science/hal-01975393}

Submitted on 9 Jan 2019

HAL is a multi-disciplinary open access archive for the deposit and dissemination of scientific research documents, whether they are published or not. The documents may come from teaching and research institutions in France or abroad, or from public or private research centers.
L'archive ouverte pluridisciplinaire HAL, est destinée au dépôt et à la diffusion de documents scientifiques de niveau recherche, publiés ou non, émanant des établissements d'enseignement et de recherche français ou étrangers, des laboratoires publics ou privés. 


\title{
THE DYNAMICS OF A TIPPE TOP*
}

\author{
A. C. $\mathrm{OR}^{\dagger}$
}

\begin{abstract}
Despite the numerous studies devoted to the dynamics of a spinning top, some disputable issues concerning the classical motion still remain unsettled. The complexity of the six degree-of-freedom motion is compounded by the assumptions that must be made about the friction law. In this paper, a numerical model based on a more general description of contact friction is developed to simulate the unusual flip behavior of an old toy, known as the Tippe top. The results appear to explain some of the puzzles raised and disputed by previous investigators.
\end{abstract}

Key words. classical rigid-body dynamics, gyroscopic motions, contact frictions, rolling, sliding

AMS subject classifications. 70E05, 70J25, 70K20, 73T05

1. Introduction. Motion of gyroscopic bodies tends to bewilder intuition. Many interesting examples described in early treatises of the subject [1], [2] still provide inspiration for modern readers. Recently, I came across a toy top that I later learnt to be the famous Tippe top (shown in Fig. 1). Unlike a regular top, this one spins on a spherical peg capable of rolling. The top has also generated numerous studies, especially in the 1950s. On the toy peg, it reads "Spin Fast! I Will Flip Over ... and Spin on the Stick!" The top appears stable when spun slowly. When spun more rapidly, however, an instability develops that leads to overturning. The top is made of a symmetric truncated wooden sphere, with a stick attached to the base of the trucated cylindrical cavity. The center of mass of the top is slightly shifted to below the sphere's center. Table 1 summarizes the measured mass property of a sample. Figure 1 also illustrates the observed sequence of tumbling motion. Such motion occurs typically over tens and hundreds of spin periods. Following the tumbling, the toy top eventually starts to spin on its stick in the reversed vertical position. This happens regardless of the type of contacting surfaces, and seems to be quite independent of initial conditions. The overturning motion is a transition from an unstable equilibrium to the stable one, but preliminary study of the motion suggests that rolling motion always remains neutrally stable, even though the center of mass can oscillate about the level plane of the center of sphere. Thus, pure rolling cannot lead to an instability. Therefore, a friction law has to be postulated in addition to the governing conservation laws. Initial conditions seem to play an unimportant role. Transitions only occur as the spin speed increases through a threshold, and the friction force destabilizes or stabilizes depending on whether the top's center of mass is below or above the sphere's center.

These observations are supported by the results of many papers of which I later became aware. A majority of these studies devoted to the Tippe top problem dated back to the 1950s, at a time when the digital computer was not widely available. Synge's [3] pioneer study attributed the reversal phenomenon to an unusual mass property of the top while his analysis assumed rolling contact. Braams [4] realized the importance of sliding friction. His analysis contains some less justifiable assumptions such as vanishing of the translational velocity at the center of mass. Hugenholtz

† Department of Mechanical, Aerospace and Nuclear Engineering and Climate Dynamics Center, Institute of Geophysics and Planetary Physics, University of California, Los Angeles, California 90024. 
TABLE 1

Mass property of the toy top.

\begin{tabular}{lc}
\hline Top Mass (oz.) & 0.64 \\
Density of wood $\left(\mathrm{oz}-\mathrm{in}^{3}\right)$ & 0.42 \\
Trans. MOI about O $\left(\mathrm{oz}-\mathrm{in}^{2}\right)$ & 0.067 \\
Trans. MOI about G $\left(\mathrm{oz}-\mathrm{in}^{2}\right)$ & 0.066 \\
Trans. MOI about C $\left(\mathrm{oz}-\mathrm{in}^{2}\right)$ & 0.220 \\
Axial MOI (oz-in $\left.{ }^{2}\right)$ & 0.066 \\
$q$, Center of mass offset & -0.094 \\
$\sigma$, Inertia ratio about $G$ & 1.011 \\
$\mu$, Dimensionless mass & 2.28 \\
1/Froude (spin at $100 \mathrm{rpm})$ & 5.53 \\
(spin at 200 rpm) & 1.38 \\
(spin at $300 \mathrm{rpm})$ & 0.62 \\
\hline
\end{tabular}

Length measures (inch) of the toy top.

\begin{tabular}{ll}
\hline Stick diameter, a & 0.25 \\
Cavity diamater, b & 0.75 \\
Cavity depth, d & 0.50 \\
C.M. height, h & 0.57 \\
Radius of sphere, R & 0.63 \\
Stick length, l & 1.00 \\
\hline
\end{tabular}

[5], followed by Parkyn [6], [7], also performed similar analyses, which confirmed the importance of eccentricity and sliding friction to the stability of the Tippe top. As later noted by Kane and Levinson [8], however, the results of [4], [7] are rather dependent on initial conditions-a property that does not seem to fit observations well. At one time, the Tippe top had attracted such attention that numerous articles were published [9]-[16]. To summarize, many of these studies recognized the importance of sliding friction, but yet most only attempted to solve the linear stability problem. O'Brien and Synge [17] argued that the simple friction law cannot account for the observed instability. Instead, the authors used a viscous friction law, which has the viscous friction varied linearly with the sliding velocity at the contact point. The authors derived the stability criterion, which shows that the top becomes unstable when exceeding a certain spin speed. Cohen [18] revisited the Tippe top problem in the 1970s and provided the first numerical simulation of the reversal motions of the top. Cohen's paper also showed a photograph of Wolfgang Pauli and Niels Bohr fascinated by the Tippe top in action. Kane and Levinson [8] conceived the Tippe top as a particular case of the more general rigid body problem and developed full numerical simulations for bodies of different shapes using the general equations of motions. However, their paper dealt with the Tippe top problem only in passing mention, although some other examples such as that of a hemispherical shell were given. Kane and Levinson also criticized O'Brien and Synge's stability condition for being insensitive to the frictional coefficient. They also criticized Cohen's model as not including adequate provisions for transitions from sliding to rolling. Indeed, Kane and Levinson's model permits a switch between rolling and sliding motions. The sliding friction according to Coulomb is $\mu_{K} N$, where $\mu_{K}$ is the coefficient of kinetic or dynamic friction and $N$ is the normal reaction. A switch to rolling is assumed to occur as the absolute contact velocity vanishes. A switch to sliding occurs when the 

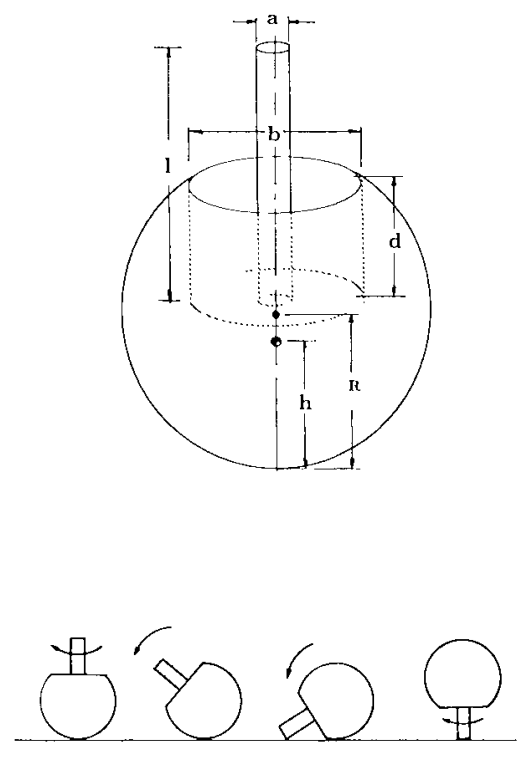

(a)

(b)

(c)

(d)

FIG. 1. Toy top configuration and tumbling sequence.

friction required to maintain rolling exceeds $\mu_{S} N$, where $\mu_{S}$ is the coefficient of static friction. Typically, the authors assume $\mu_{K}$ to be slightly less than $\mu_{S}$ to permit the mechanism to work. Like Cohen, however, Kane and Levinson do not include the viscous term in their friction law. For the class of problems, the contact friction is extremely important, and it dictates the motions. Descriptions of friction laws are available in the literature since the last century. For references, refer to Routh [19] and the references in Cohen [18].

The aim of this paper is to provide a direct solution to the Tippe top motion, which may serve to summarize previous findings and to resolve the puzzles that still remain. Indeed, it becomes clear in the following that both viscous and Coulomb frictions can cause the transition to reversal. For the case of the Tippe top, a transition from sliding to rolling never seems to occur. For this purpose, our model uses a general frictional law that includes both Coulomb and the viscous terms, with sliding being assumed throughout. Along with the sliding model, the model based on the assumption of pure rolling is also simulated, to provide a comparison between the two cases. Section 2 contains the formulation of the problem. Section 3 provides the linear stability analysis. Section 4 contains the full numerical solution of the nonlinear problem. In $\S 5$, we conclude with some further remarks.

2. Formulation. Governing equations. We refer to Fig. 2 for the geometry of spherical top. We use a nonrotating Cartesian frame attached to the sphere's center $O, \mathcal{F}_{a}$, which is defined by a triple of unit vectors, $(\vec{I}, \vec{J}, \vec{K})^{T}(T$ denotes transpose of a column vector). A second Cartesian frame fixed rigidly to the body, $\mathcal{F}_{b}$, is also used. This frame's axes are defined by a second triple of unit vectors, $(\vec{i}, \vec{j}, \vec{k})^{T}$, where the axes align with the body's principal axes. The vectrix notation [20] is used here for developing the governing equations from a vectorial form. In this notation, an 


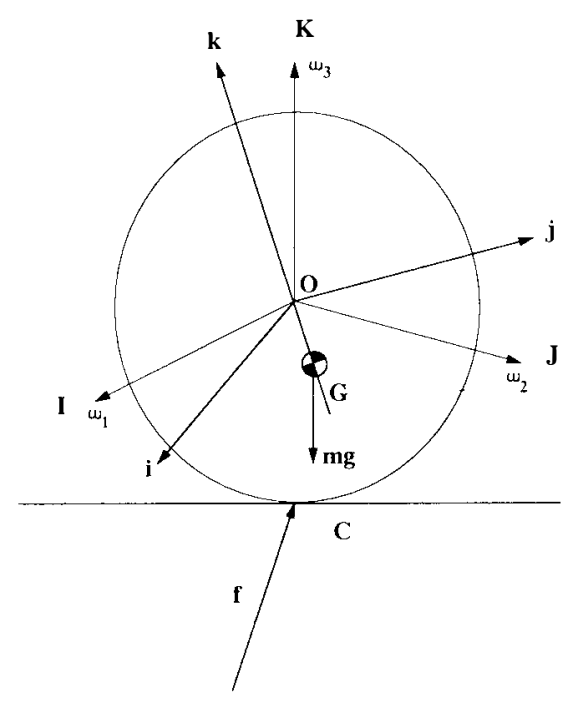

FIG. 2. Spherical model configuration.

abstract vector $\vec{v}$ is expressed as

$$
\vec{v}=\mathcal{F}_{a}^{T} \mathbf{V}=\mathcal{F}_{b}^{T} \mathbf{v}
$$

where the upper (lower) case boldface variable refers to the column vector relative to $\mathcal{F}_{a}\left(\mathcal{F}_{b}\right)$, that is, $\mathbf{V}=\left(V_{1}, V_{2}, V_{3}\right)^{T}$ and $\mathbf{v}=\left(v_{1}, v_{2}, v_{3}\right)^{T}$. The cross product between two vectors $\vec{u} \times \vec{v}$ is defined as $\mathcal{F}_{a}^{T} \mathbf{U}^{\times} \mathbf{V}$ or, equivalently, $\mathcal{F}_{b}^{T} \mathbf{u}^{\times} \mathbf{v}$. For a column vector $\mathbf{u}=\left(u_{1}, u_{2}, u_{3}\right)^{T}, \mathbf{u}^{\times}$is the skew-symmetric matrix

$$
\mathbf{u}^{\times}=\left(\begin{array}{ccc}
0 & -u_{3} & u_{2} \\
u_{3} & 0 & -u_{1} \\
-u_{2} & u_{1} & 0
\end{array}\right) .
$$

To reduce the number of parameters, we introduce the spin period $\Omega^{-1}$ as the timescale and the radius $R$ as the lengthscale. The linear and angular momentum equations referring to $\mathcal{F}_{a}$ are then

$$
\begin{gathered}
\mu\left(\dot{\mathbf{V}}_{0}+q \ddot{\mathbf{k}}\right)=\mathbf{F}-\mu F r^{-1} \mathbf{K}, \\
\frac{d}{d t}\left(\mathbf{I}_{\mathbf{0}} \boldsymbol{\omega}\right)=\mu q \dot{\mathbf{V}}_{0}^{\times} \mathbf{k}-\mu q F r^{-1} \mathbf{k}^{\times} \mathbf{K}-\mathbf{K}^{\times} \mathbf{F},
\end{gathered}
$$

where $\mathbf{I}_{\mathbf{0}}$ is the moment of inertia tensor about point $O ; \mathbf{V}_{\mathbf{0}}$ is the absolute velocity at point $O ; \boldsymbol{\omega}$ is the angular velocity, $\mathbf{k}$ is the unit vector along the top axis; $\mathbf{F}$ is the force acting on the body through the contact point. The major nondimensional parameters are the scaled top mass $\mu$, the Froude number $F r$, the inertia ratio $\sigma$, and the initial center of mass offset $q$. The nondimensional parameters are defined as

$$
\mu=\frac{M R^{2}}{A_{0}}, \quad F r=\frac{\Omega^{2} R}{g}, \quad \sigma=\frac{C}{A_{0}},
$$


where $M$ is the body mass; $g$ is the gravity acceleration; $A_{0}$ and $C$ are, respectívely, principal moments of inertia about point $O$. The equilibrium solution of the initial state is

$$
\mathbf{F}=\mu F r^{-1} \mathbf{K}, \quad \boldsymbol{\omega}=\Omega \mathbf{K}, \quad \mathbf{V}_{0}=0, \quad \mathbf{k}=\mathbf{K} .
$$

We further introduce three direction cosine parameters $l, m, n$ for the attitude, to facilitate the equations to be expressed in the component form. The direction cosines satisfy identities

$$
\mathbf{k}=(l, m, n)^{T}, \quad l^{2}+m^{2}+n^{2}=1 .
$$

It can be easily checked that $\mathbf{I}_{\mathbf{0}}$ has an expression

$$
\mathbf{I}_{\mathbf{0}}=\mathbf{1}_{\mathbf{3}}+(\sigma-1) \mathbf{k k}^{T},
$$

where $\mathbf{1}_{\mathbf{3}}$ is the order-3 identity matrix. The transverse axes have a degenerate orientation due to axisymmetry. The kinematics are governed by

$$
\dot{\mathbf{k}}=\omega^{\times} \mathbf{k} \text {. }
$$

Equations (1), (2), (4) complete the twelfth-order six degree-of-freedom dynamical problem with the specification of $\mathbf{F}$. The translational displacement $\mathbf{X}_{\mathbf{0}}$ is not coupled into the dynamics and can be determined from

$$
\dot{\mathbf{X}}_{0}=\mathbf{V}_{\mathbf{0}} \text {. }
$$

In the case of a pure rolling, the velocity at the moving contact point vanishes identically throughout the motion, that is,

$$
\mathbf{V}_{\mathbf{c}}=\mathbf{0}, \quad \mathbf{V}_{\mathbf{c}}=\mathbf{V}_{0}+\mathbf{K}^{\times} \boldsymbol{\omega} .
$$

The rolling constraint permits $\mathbf{F}$ to be eliminated from combining the translational and rotational equations. In general, the force at contact point $\mathbf{F}$ has the following representation:

$$
\mathbf{F}=N \mathbf{K}+\mathbf{f}
$$

where $N$ is the normal reaction and $\mathbf{f}$ is the tangential force. While $N$ can be determined from the normal component of the dynamical equations, $\mathbf{f}$ must be supplied independently. In any case, it is known that $\mathbf{f}$ opposes motion. In a dimensional form, $\mathbf{f}$ is given by

$$
\mathbf{f}=-N\left(\mu_{K}+\mu_{v} V_{c}+\ldots\right) \mathbf{e}_{\mathbf{v}}
$$

where $\mu_{v}$ is the coefficient of viscous friction; $V_{c}$ is given by $\mathbf{V}_{\mathbf{c}}=V_{c} \mathbf{e}_{\mathbf{v}}$. The above expression is a generalization of the Coulomb friction law to include the viscous term. The Coulomb's dynamic law the corresponds to the following truncated expression:

$$
f=-\mu_{K} N
$$

The next term in (6) is proportional to the sliding velocity, which is similar to viscous friction. In nondimensional form, the friction law becomes

$$
f=-\left(\alpha_{K}+\alpha_{v} V_{c}\right),
$$

where now the Coulomb parameter $\alpha_{K}$ and the viscous parameter $\alpha_{v}$ are given by

$$
\alpha_{K}=\mu F r^{-1} \mu_{K}, \quad \alpha_{v}=\mu \Omega R F r^{-1} \mu_{v} .
$$


3. The results. (i) Stability. The component form of our governing equations is shown in the Appendix. O'Brien and Synge's stability condition appears qualitatively correct, but the dependence of the stability on the friction coefficient is not included. To understand the roles of various parameters, the stability problem will be briefly discussed before we turn to the numerical sliding model. First, we consider the two classical examples of a top with a fixed-pivot and a rolling peg. Stability conditions similar to those given below can be found in [1], [2]. Introducing time dependence of $e^{i \lambda t}$, we obtain the characteristic roots after simple manipulation of the governing equations,

$$
\lambda=\left(\frac{\hat{\sigma}}{2}-1\right) \pm\left(\frac{\hat{\sigma}^{2}}{4}-\mu F r^{-1} h\right)^{1 / 2},
$$

where $\hat{\sigma}$ is the axial to transverse moments of inertia ratio about the contact point, and

$$
h= \begin{cases}q & \text { for a rolling top } \\ 1+q & \text { for a fixed-pivot top. }\end{cases}
$$

The stability condition is

$$
1>4 \mu F r^{-1} h / \hat{\sigma}^{2}
$$

and a rolling top always satisfies this condition since $q<0$. A rolling top also possesses two integrals of motion (a fixed-pivot top has three integrals). One is the total energy. The other is the angular momentum projected on to the line connecting the contact point $C$ to the center of mass $G$, as there is no moment applied about this line. This angular momentum $h_{c g}$ is given by

$$
h_{c g}=\mathbf{r}_{\mathrm{cg}}^{T}\left(\mathbf{I}_{\mathbf{g}}+\mu\left(\mathbf{r}_{\mathbf{c g}}^{T} \mathbf{r}_{\mathrm{cg}} \mathbf{1}_{\mathbf{3}}-\mathbf{r}_{\mathbf{c g}} \mathbf{r}_{\mathbf{c g}}^{T}\right)\right) \boldsymbol{\omega}, \quad \mathbf{r}_{\mathbf{c g}}=q \mathbf{k}+\mathbf{K}
$$

Next, we consider the sliding case. As remarked by Kane and Levinson [8], the linear stability criterion of O'Brien and Synge [17] is inadequate, since it does not depend on the friction coefficient. We only need to focus on the case of $\alpha_{K}=0$ and $\alpha_{v} \neq 0$. The Coulomb term is nonlinear. The subsequent numerical simulations will show that this term alone can also produce instability, but with an algebraic rather than exponential growth rate. We let $\omega_{3}=n=1, \omega=\omega_{1}+i \omega_{2}, \Phi=l+i m$, and $V=V_{1}+i V_{2}$ and we obtain the following linearized set:

$$
\begin{aligned}
\dot{\omega}+i \mu q \dot{V} & =i\left(-(\sigma-1)+\mu q F r^{-1}\right) \Phi+\left(-\alpha_{v}+i(\sigma-1)\right) \omega+i \alpha_{v} V \\
\dot{\Phi} & =i \Phi-i \omega \\
\dot{V}-i q \dot{\omega} & =q \Phi-\left(q+i \frac{\alpha_{v}}{\mu}\right) \omega-\frac{\alpha_{v}}{\mu} V
\end{aligned}
$$

The problem depends on the parameters $\mu, q, F r, \sigma$, and $\alpha_{v}$. The growth rate and frequency dependence on these parameters will be discussed only in cases relevant to the physical problem. Figures 3(a) and 3(b) show the change of growth rate and frequency, respectively, with respect to the center-of-mass offset $q$ for different $\mathrm{Fr}^{-1}$ (marked alongside curves) at $\sigma=1.0, \mu=2.28$, and $\alpha_{v}=0.5$. We see that a weak spin relative to gravity (i.e., small $F r$ ) is stabilizing if the center of mass is below $O$ and destabilizing if it is above $O$. A reversal of this stability property occurs at high $\mathrm{Fr}$. The inertia ratio $\sigma$ also affects the stability property, as suggested by 
Figs. 3(c) and 3(d) ( $\sigma$ marked alongside curves). In the limit of very strong spin or very weak gravity, i.e., $\mathrm{Fr}^{-1}=0$, the inertia ratio stabilizes the top above the value of $\sigma=1.14$. The result is consistent with the well-established principle that the stability of a body depends on the shape of its moment of inertia ellipsoid, if energy dissipation is permitted (see also [4]). An oblate spheroid $(\sigma>1)$ indicates stability, while a prolate spheroid $(\sigma<1)$ indicates instability. To show the dependence of the growth rate on the friction coefficient $\alpha_{v}$, we now consider the case where $F^{-1}=0$ and $\sigma=1$. Figure 4 shows a family of growth-rate curves versus $q$. The solid curves rise with increasing $\alpha_{v}$ to 0.1 to 1.0. For this branch, a stronger friction means more destabilization. The family of curves has a maxima with respect to $\alpha_{v}$, however, after which further increase in friction leads to a decrease in growth rate. The second branch corresponds to $\alpha_{v}=2.0$ and 5.0 (dotted lines). Thus, while a moderate amount of friction destabilizes, a stronger friction tends to destabilize.

(ii) Numerical simulation. The full sliding model (see Appendix for the full equations) is now simulated using a fourth-order Runge-Kutta scheme. The initial condition is such that $\omega_{3} \approx 1$ and $n \approx 1$, otherwise arbitrary. The parameters used resemble that of the actual top. First, we investigate the case for vanishing $\alpha_{K}$ and

(a)

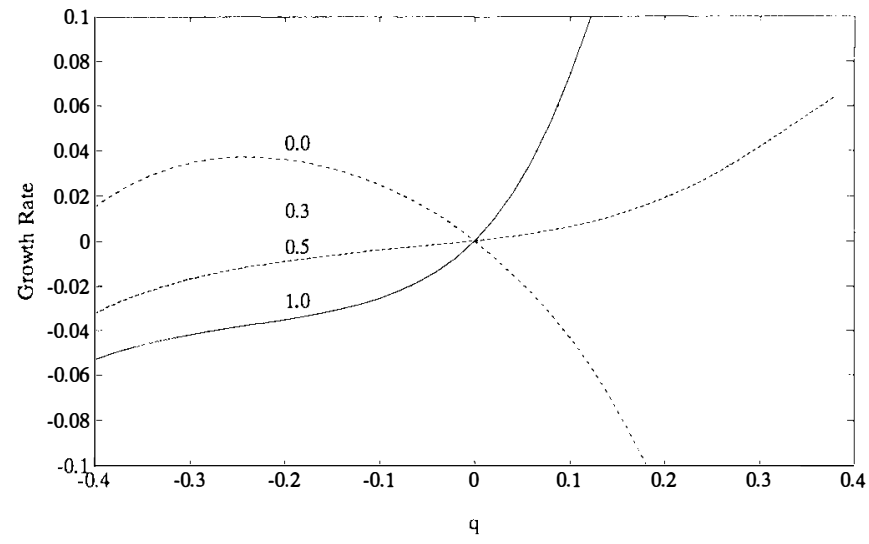

(b)

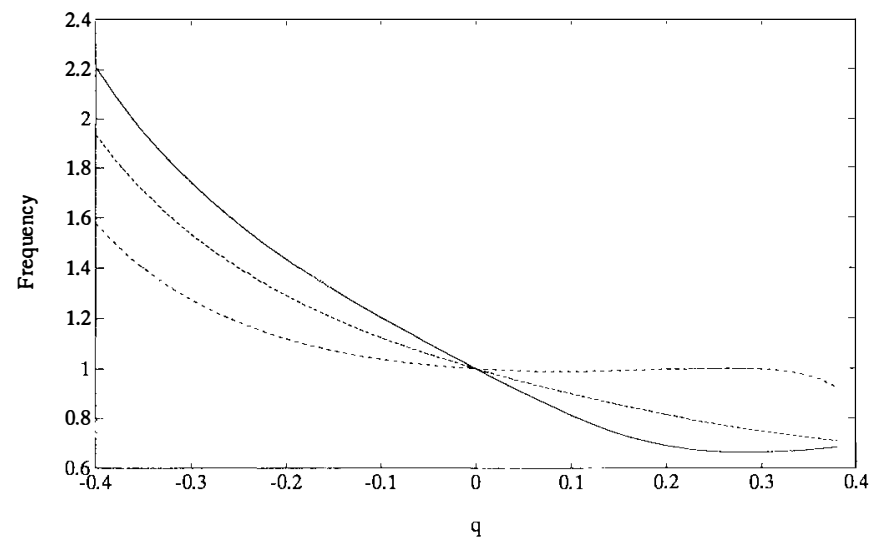

FIG. 3. Stability plots showing (a) growth rate; (b) frequency for $\sigma=1$ and varying $\mathrm{Fr}^{-1}$; (c) growth rate; (d) frequency for $\mathrm{Fr}^{-1}=0$ and varying $\sigma$. 

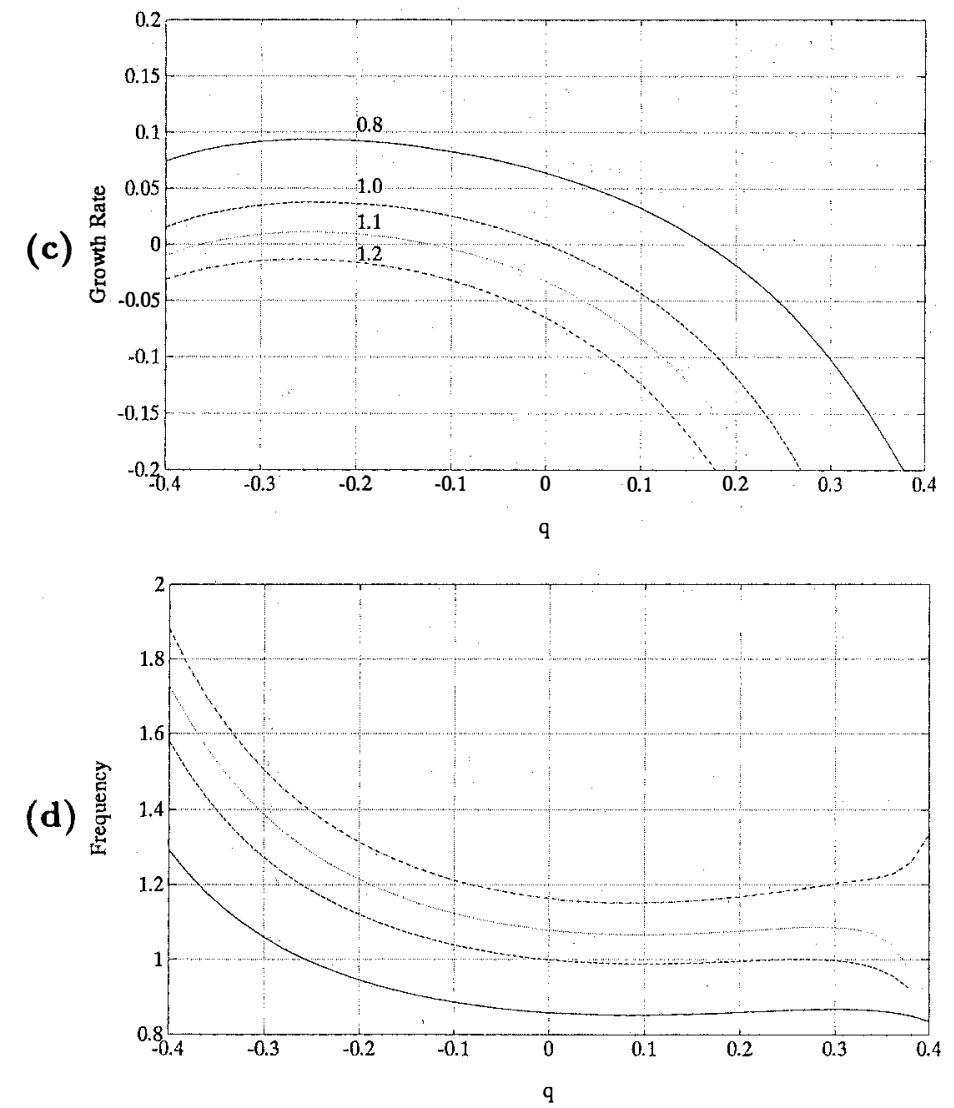

FIG. 3 (continued).

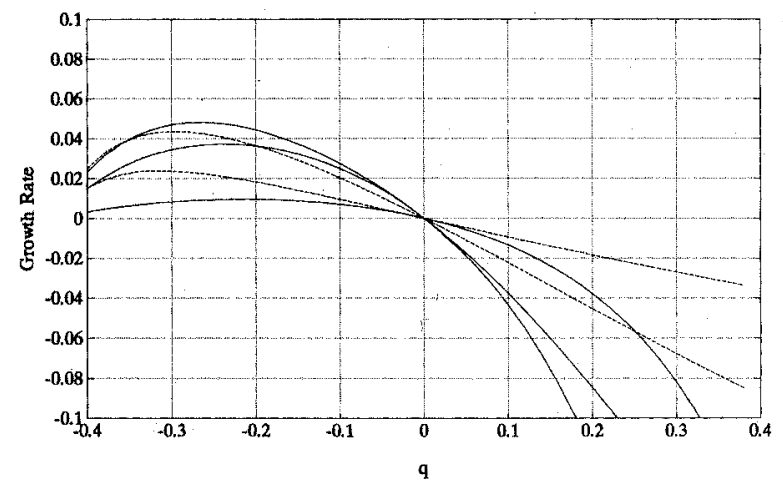

FIG. 4. Stability plot showing growth rate with varying friction parameter, for $\mathrm{Fr}^{-1}=0$ and $\sigma=1$ 
finite $\alpha_{v}$ and later we examine the effect of finite $\alpha_{K}$. The simulation corresponds to $\alpha_{v}=0.5, \mu=2.28, q=-0.1$, and $\sigma=1.0$. We present two solutions in Fig. 5, which correspond to $F r^{-1}=0.3$ and $F r^{-1}=0.1$ (marked alongside curves). With the top's radius of 0.6366 inch, the parameter values correspond to a spin speed of 7.15 and 12.4 revolutions per second, respectively. For the low $\mathrm{Fr}$ case, the initial conditions are $\omega_{1}=0.0219, \omega_{2}=0.0047, \omega_{3}=-0.9997, l=0.0676, m=0.0676, n=0.9954$, $V_{1}=0.0935$, and $V_{2}=0.0384$. For the high $F r$ case, we have $\omega_{1}=0.00219$, $\omega_{2}=0.0047, \omega_{3}=-0.9999, l=0.0080, m=0.0150, n=0.9998, V_{1}=0.0110$, and $V_{2}=0.0038$. Figure 5 (a) shows that there is a drop of spin speed after reversal due to a loss of energy and the work done in raising the center of mass. Figure 5(b) shows the magnitude of transverse velocity (note that all components are measured with respect to $\mathcal{F}_{a}$ ). Where the top axis passes through a polar angle of $90^{\circ}$, the transverse angular speed only peaks at a small value of about 0.12 . Figure 5(c) shows the polar angle of the top's overturning axis, measured from the upward vertical. The crossing at the $90^{\circ}$ plane occurs at about 78 and 41 spin periods from the start, or 10.9 seconds and 3.3 seconds after spun off. For the case of $\mathrm{Fr}^{-1}=0.3$, we further show the components of energy. Figure 6 indicates that the translational energy at $G$ is very small. Even the potential energy is significantly smaller than the spin energy (about $G$ ) for this low $F r$ case. The dissipation of energy is quite noticeable. The axial and transverse angular momenta are quite similar to the angular velocities, which will not be shown. For the same simulation, we show in Fig. 7 the translational displacement of the sphere's center of mass. Along the locus, the translational velocity vector is indicated by arrows at a regular interval of $5 /(2 \pi)$ spin-period. In the enlarged portion of the curve, the arrows are shown at intervals of $1 /(2 \pi)$. In Fig. 8 , a three-dimensional view of motion of the axis is shown by tracking $\mathbf{k}$ on a unit sphere at uniform intervals of $1 /(2 \pi)$ (cross marks). The axis spirals outward from the north pole and inward to the south pole.

Finally, we examine the friction effect. For the same case with $\alpha_{K}=0, \alpha_{v}=0.5$, and $\mathrm{Fr}^{-1}=0.3$, we show a comparison between the magnitude of friction force according to the sliding model (solid) and to the rolling model (solid lines) in Fig. 9(a). Since $\alpha_{K}=0$, the sliding friction is thus proportional to the contact velocity $V_{c}$, and therefore the sliding curve can be interpreted as $V_{c}$ apart from a multiplication constant. The friction required for maintaining rolling appears fairly stationary. However, during the tumbling process, the sliding friction typically exceeds the rolling friction. Toward the final equilibrium, a transition from sliding to rolling may occur, as the contact speed tends to zero. In Fig. 9(b), we show the tumbling effect for four different cases of friction, in terms of the polar angle and at $F r^{-1}=0.3$. The same parameters and initial conditions as before apply, and the friction coefficients used are (1) $\alpha_{K}=0, \alpha_{v}=0.5 ;$ (2) $\alpha_{K}=0.5, \alpha_{v}=0.5$; (3) $\alpha_{K}=0.5, \alpha_{v}=0.1$; (4) $\alpha_{K}=0.5, \alpha_{v}=0.0$. In the last case, viscous friction is absent. The result shows that tumbling can still occur but appears considerably weaker. The destabilizing role of the Coulomb friction becomes clear if a Fourier expansion of the Coulomb term is performed. The expansion contains a sinusoidal forcing that has the same sign as the viscous friction. The Coulomb forcing yields an algebraic growth, in contrast to the viscous forcing that yields an exponential growth. In reality, it is anticipated that both Coulomb and viscous frictions are present. Thus cases (2), (3) are more relevant. One last debatable issue is whether transitions between rolling and sliding are possible during the course of the motion. In all the simulations we studied, the magnitude of the contact velocity hardly ever vanishes. As an example, we show the sliding speed 
(a)

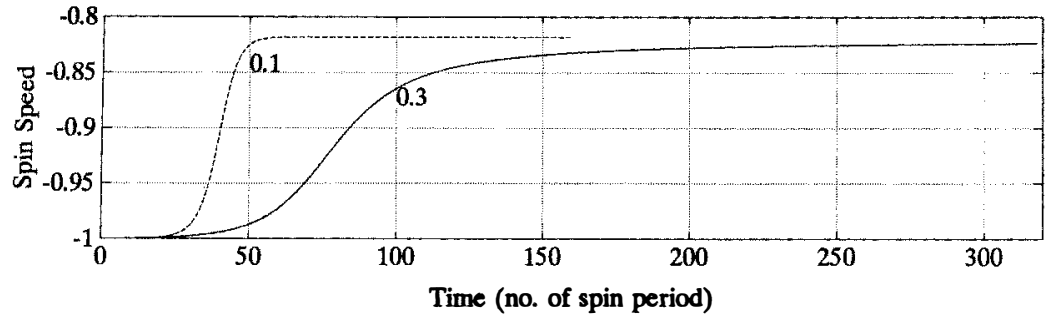

(b)

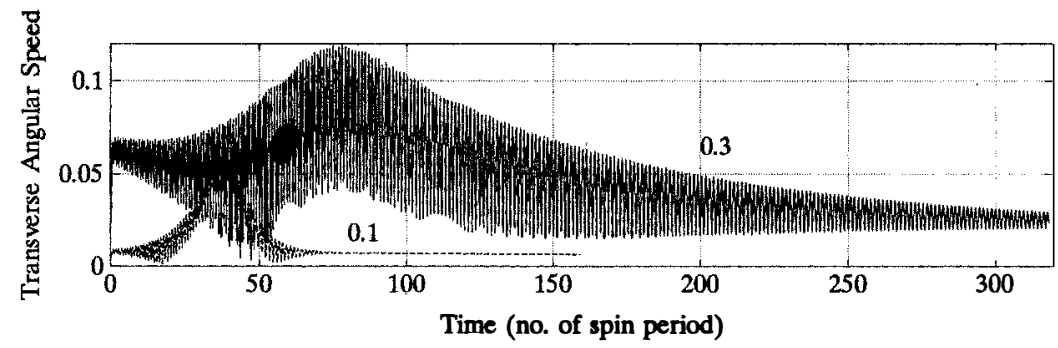

(c)

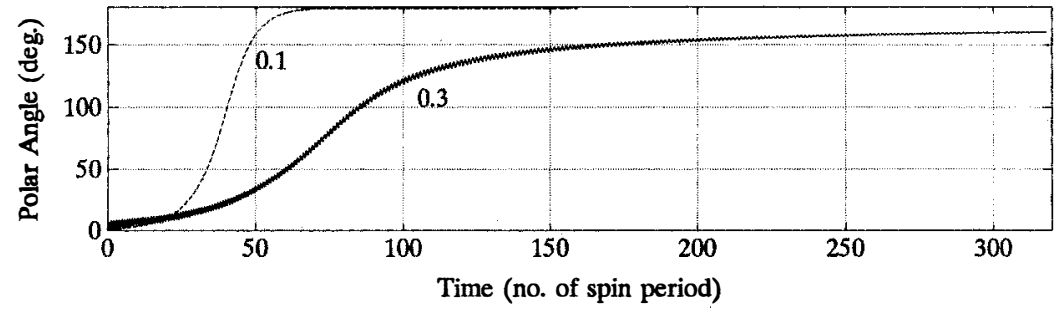

FIG. 5. Nonlinear simulations showing the (a) spin speed; (b) transverse angular rate; (c) polar angle in time for the cases $\mathrm{Fr}^{-1}=0.1$ and 0.3 .

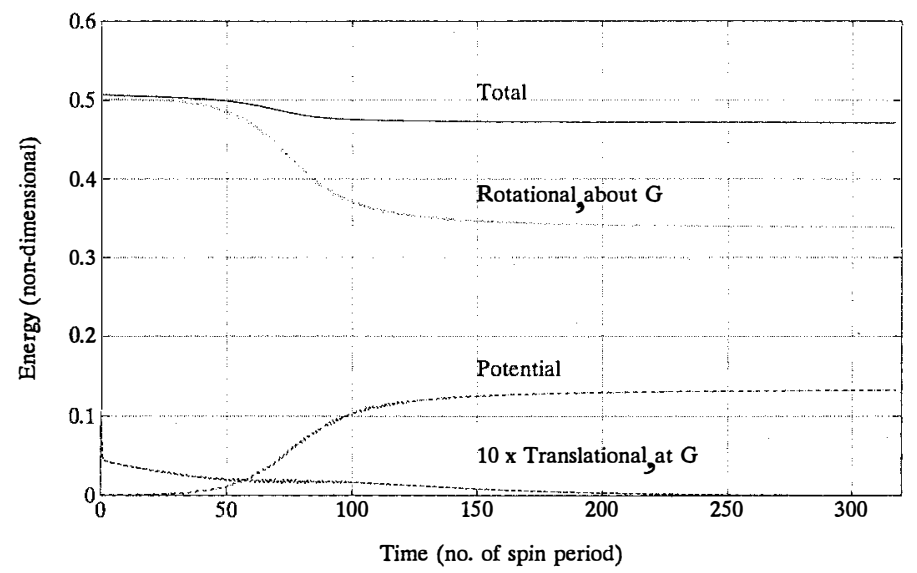

FIG. 6. The top's energy components in time. 


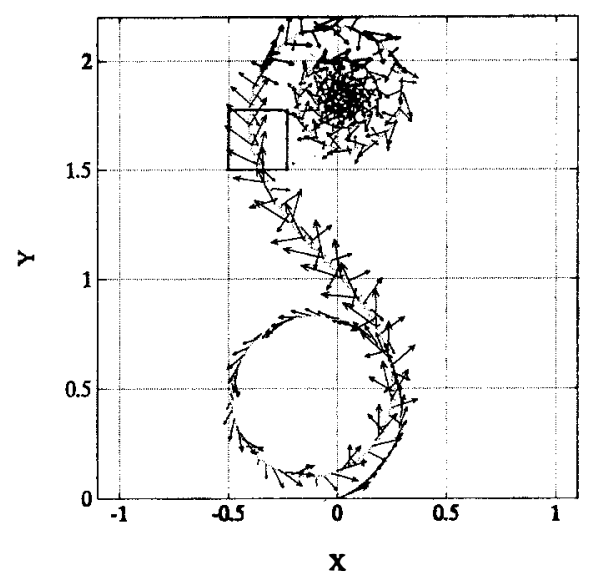

(a)

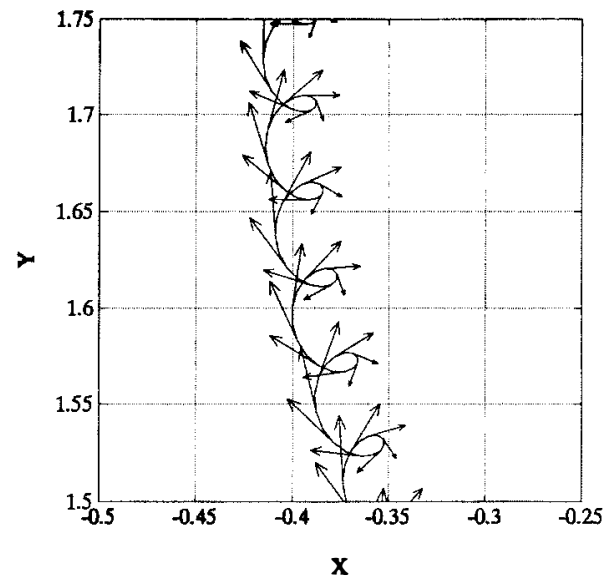

(b)

FIG. 7. The locus of the center of sphere. (a) Translation of the sphere center. (b) Magnified view of the translation.

\section{A 3D View of the Orientation of the Top Axis}

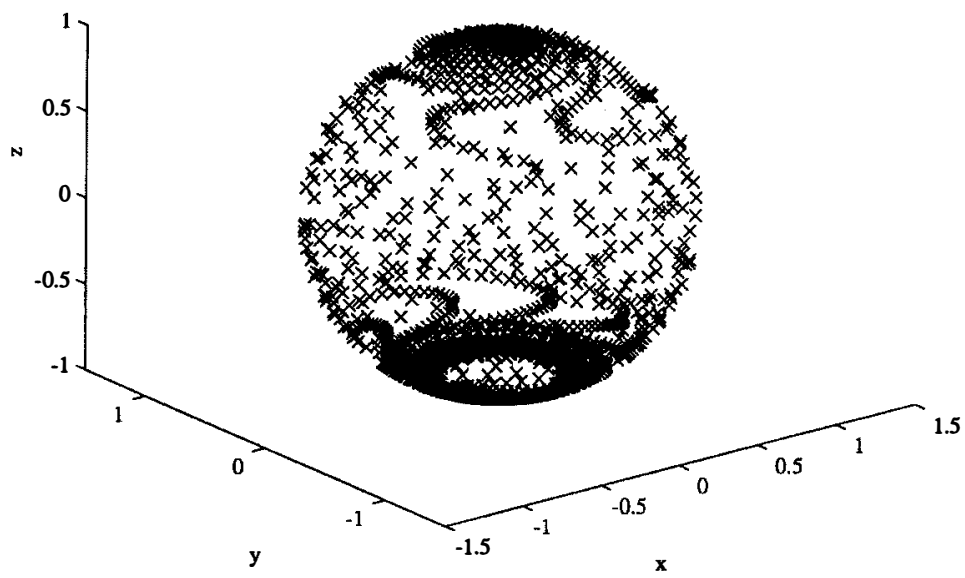

FIG. 8. A three-dimensional view showing the excursion of the sphere's axis during overturn.

for case (4) in Fig. 9(c). The result suggests that a switch between sliding and rolling is not an important ingredient for our analysis.

4. Concluding remarks. The complete sequence of tumbling motions of the Tippe top between the two vertical spin equilibria is simulated numerically using a spherical model with an inertia tensor close to the actual top's. Several important points concerning the effects of friction are noted. (i) The viscous friction was first introduced in [1] to produce instability of the spin equilibrium. In fact, the Coulomb 
(a)

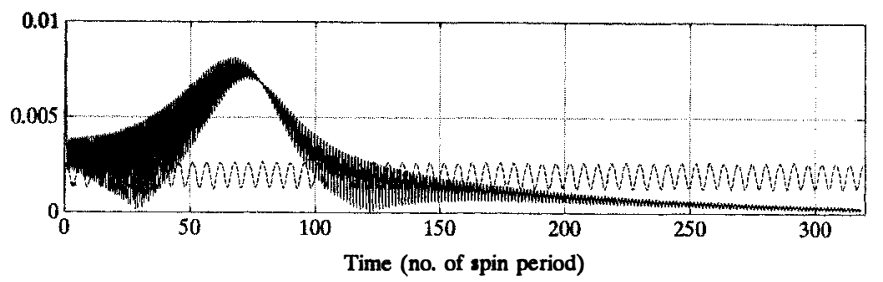

(b)

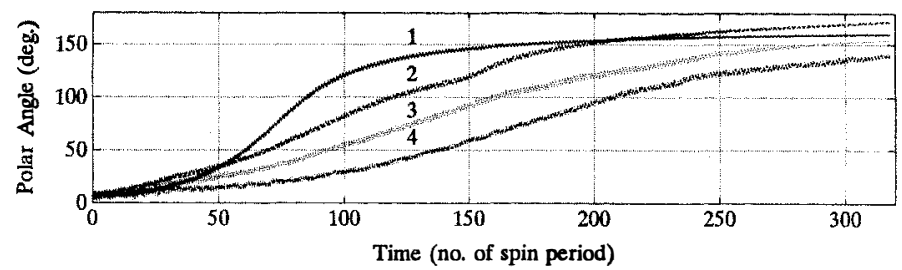

(c)

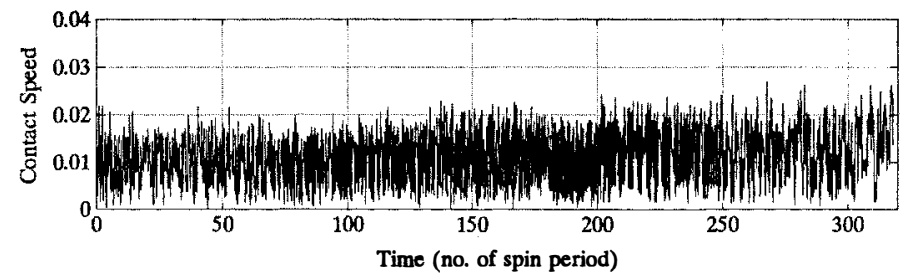

FIG. 9. Time plots to illustrate the effect of changing frictional coefficients: (a) sliding friction (solid) and friction according to the rolling model (dotted); (b) polar angle change; (c) sliding speed for the case of zero $\alpha_{v}$.

friction can also destabilize. It produces an initial growth that is algebraic rather than exponential. (ii) A linear stability analysis based on the viscous friction indicates that the magnitude of the friction coefficient is important to both the growth rate and stability limit. (iii) The phenomenon of the Tippe top appears to be almost exclusively sliding. Once sliding is established, there is hardly ever any switching to rolling motion.

Appendix. The governing equations in a scalar form. The angular momentum equations are

$$
\begin{aligned}
\dot{\omega}_{1}+ & (\sigma-1) l \dot{\omega}_{3}+\mu q\left(-n \dot{V}_{2}+m \dot{V}_{3}\right) \\
& =(\sigma-1)\left(-\omega_{2}+m\right) \omega_{3}-\mu q F r^{-1} m+f_{2}, \\
\dot{\omega}_{2}+ & (\sigma-1) m \dot{\omega}_{3}+\mu q\left(n \dot{V}_{1}-l \dot{V}_{3}\right) \\
& =(\sigma-1)\left(\omega_{1}-l\right) \omega_{3}+\mu q F r^{-1} l-f_{1}, \\
\sigma \dot{\omega}_{3}+ & (\sigma-1)\left(l \dot{\omega}_{1}+m \dot{\omega}_{2}\right)+\mu q\left(-m \dot{V}_{1}+l \dot{V}_{2}\right) \\
& =(\sigma-1)\left(\left(-\omega_{2}+m\right) \omega_{1}+\left(\omega_{1}-l\right) \omega_{2}\right),
\end{aligned}
$$

where $f_{1}$ and $f_{2}$ are the traction force. The attitude equations are

$$
\dot{l}=-\omega_{3} m+\omega_{2} n, \quad \dot{m}=\omega_{3} l-\omega_{1} n, \quad \dot{n}=-\omega_{2} l+\omega_{1} m .
$$


The translational momentum equations are

$$
\begin{aligned}
\dot{V}_{1}+q\left(n \dot{\omega}_{2}-m \dot{\omega}_{3}\right) & =q\left(\left(\omega_{3}^{2}+\omega_{2}^{2}\right) l-\omega_{1} \omega_{2} m-\omega_{1} \omega_{3} n\right)+\frac{1}{\mu} f_{1} \\
\dot{V}_{2}-q\left(n \dot{\omega}_{1}-l \dot{\omega}_{3}\right) & =q\left(\left(\omega_{3}^{2}+\omega_{1}^{2}\right) m-\omega_{1} \omega_{2} l-\omega_{2} \omega_{3} n\right)+\frac{1}{\mu} f_{2} \\
\dot{V}_{3}+q\left(m \dot{\omega}_{1}-l \dot{\omega}_{2}\right) & =q\left(\left(\omega_{1}^{2}+\omega_{1}^{2}\right) n-\omega_{1} \omega_{3} l-\omega_{2} \omega_{3} m\right)+\frac{1}{\mu} N-F r^{-1}
\end{aligned}
$$

where $N$ is the normal force. The velocity at the point of contact is given by

$$
V_{c 1}=V_{1}-\omega_{2}, \quad V_{c 2}=V_{2}+\omega_{1}, \quad V_{c 3}=0 .
$$

Since the top stays in contact with the horizontal surface, we can eliminate the vertical translational momentum equation. Thus $N$ is determined the vertical kinemtic constraint. In the rolling model, we can eliminate $f_{1}$ and $f_{2}$ from the two set of momentum equations. Then (15) is used to eliminate the translational velocity. The three remaining equations, together with the attitude equations form the rolling model. For the sliding model, (12)-(14) are solved in conjunction with the following friction law:

$$
f_{1}=-\alpha_{K} \operatorname{sgn}\left(V_{c 1}\right)-\alpha_{v} V_{c 1}, \quad f_{2}=-\alpha_{K} \operatorname{sgn}\left(V_{c 2}\right)-\alpha_{v} V_{c 2} .
$$

Acknowledgments. I thank the anonymous referees, particularly Dr. J.R. Ockendon, for numerous suggestions and for the source of references. Also, this work would not have been completed without the inspiration of the toy top.

\section{REFERENCES}

[1] H. Crabtree, An Elementary Treatment of the Theory of Spinning Tops and Gyroscopic Motion, Chelsea, New York, 1967.

[2] J. PERRY, Spinning Tops and Gyroscopic Motions, Dover, New York, 1957.

[3] J. L. SYNGE, On a case of instability produced by rotation, Philos. Mag., 43 (1952), pp. 724-728.

[4] C. M. BraAms, On the influence of friction on the motion of a top, Physica, 18 (1952), pp. 503-514.

[5] N. M. Hugenholtz, On tops rising by friction, Physica, 18 (1952), pp. 515-527.

[6] D. G. PARKYN, The rising of tops with rounded pegs, Physica, 24 (1958), pp. 313-330.

[7] — The inverting top, Math. Gaz., 40 (1956), pp. 260-265.

[8] T. R. Kane And D.A. Levinson, A realistic solution of the symmetric top problem, J. Appl. Mech., 45 (1978), pp. 903-909.

[9] A. D. FOKKER, The tracks of tops' pegs on the floor, Physica, 18 (1952), pp. 497-502.

[10] C. M. BRAAMS, The symmetrical spherical top, Nature, 170 (1952), p. 31.

[11] J. A. JACOBS, Note on the behavior of a certain symmetrical top, Amer. J. Phys., 20 (1952), pp. 517-518.

[12] C. M. BraAms, The tippe top, Amer. J. Phys., 22 (1954), p. 568.

[13] A. R. Del Campo, Tippe top (topsy-turnee top) continued, Amer. J. Phys., 23 (1955), pp. 544545.

[14] I. M. Freeman, The tippe top again, Amer. J. Phys., 24 (1956), p. 178.

[15] J. B. HART, Angular momentum and tippe top, Amer. J. Phys., 27 (1959), p. 189.

[16] F. F. Johnson, The tippy top, Amer. J. Phys., 28 (1960), pp. 406-407.

[17] S. O'Brien Ánd J.L. Synge, The Instability of the Tippe-Top Explained by Sliding Frictıon, Proc. of the Royal Irish Academy, Vol. 56, Section A, Feb. 1954, pp. 23-35.

[18] R. J. Cohen, The tippe top revisited, Amer. J. Phys., 45 (1977), pp. 12-17.

[19] E. J. Routh, Dynamics of a System of Rigid Bodies, Macmillan, New York, 1905.

[20] P. C. Hughes, Spacecraft Attitude Dynamics, John Wiley, New York, 1986. 